\title{
Patient activation is associated with fewer visits to both general practice and emergency departments: a cross-sectional study of patients with long-term conditions
}

\author{
Authors: Isaac Barker, Adam Steventon and Sarah Deeny
}

\section{Aims}

Patient activation refers to the knowledge, skills and confidence that a person has to manage their health. We assessed the association between patient activation and the utilisation of primary and secondary care, for patients with long-term health conditions (LTCs) registered in Islington CCG, a cosmopolitan inner London area.

\section{Methods}

The 13-question patient activation measure (PAM) questionnaire was sent by post (in October 2015 and 2016) to all patients over 18 years of age with common long-term conditions. This was then returned by post, by a patient to their GP. We analysed the patient record of those patients with a PAM score recorded on their medical record. For each patient, we summarised their numbers of general practice contacts, accident and emergency (A\&E) visits, emergency admissions, elective admissions, total inpatient admissions, and outpatient appointments between January 2013 and April 2016. For each category of healthcare utilisation, we fitted regression models to estimate its association with the PAM score (which ranges from 1 to 100). Models controlled for demographic (age, deprivation and sex) and clinical (type and number of comorbidities) patient characteristics and allowed for between-practice variation.

\section{Results}

We obtained linked records for 10,427 patients from 34 general practices. When modelled, controlling for other factors, a 20-point increase in PAM was associated with 9.05\% fewer GP contacts (95\% CI, 0.89-0.93), 20.90\% fewer A\&E attendances (95\% CI, 0.75-0.83) and 23.26\% fewer emergency admissions (95\% CI, 0.71-0.83) per person. PAM was not significantly associated with the number of outpatient attendances, elective inpatient admissions, or total inpatient admissions.

\section{Conclusions}

We found that less activated patients had higher utilisation of primary and emergency care. While we accounted for the type of LTC and number of comorbidities, further research is needed to determine the interaction between the severity of LTC, PAM and access to care. By tailoring healthcare management strategies to the activation level of the patient, physicians may be able to 'meet patients where they are' and reduce reliance on emergency care.

\section{Conflict of interest statement}

No conflicts of interest. 